Breast Care 2016;11:381

The editors of BREAST CARE gratefully acknowledge the committment and advice of the associate editors and guest editors in deciding which topics to pursue, which manuscripts to publish, and what modifications to make to ensure medical and scientific accuracy and suitability for our readers.

\title{
Guest Editors
}

Carsten Denkert, Berlin

Peter Fasching, Erlangen

Tanja Fehm, Düsseldorf

Volker Möbus, Frankfurt/M.

Florian Schütz, Heidelberg

Isabell Witzel, Hamburg

Furthermore, the editors extend their gratitude and appreciation to the following reviewers whose comments and criticism ensure the quality of the articles published in this journal.

\section{Reviewers}

Oshaani Abeyakoon, Cambridge Werner Bader, Bielefeld Rajendra Badwe, Mumbai Zsuzsanna Bago-Horvath, Vienna Pascal A. Baltzer, Vienna Rupert Bartsch, Vienna Freerk T. Baumann, Cologne Hubert Bickel, Vienna Joachim Bischoff, Dessau Vesna Bjelic-Radisic, Graz Jens-Uwe Blohmer, Berlin Paola Clauser, Udine Barbara Collins, Ottawa Serban-Dan Costa, Magdeburg Theresa Czech, Innsbruck Silvia Darb-Esfahani, Berlin Amelie de Gregorio, Ulm Alexander de Vries, Feldkirch Max Dieterich, Rostock Timm Dirrichs, Aachen Christoph Domschke, Heidelberg Daniel Egle, Innsbruck Nagi El Saghir, Beirut Ruth Exner, Vienna Hermann Faller, Würzburg Tanja Fehm, Tübingen Cornelia Focke, Neubrandenburg Etelka Földi, Hinterzarten Pierfrancesco Franco, Torino Carlo Fremd, Heidelberg Michael Friedrich, Krefeld Oleg Gluz, Mönchengladbach
Eva-Maria Grischke, Tübingen Eva Gross, Munich Friederike Hagemann, Münster Annette Hasenburg, Mainz Guido Henke, St. Gallen Arnd Hönig, Mainz Michael Hubalek, Innsbruck Barbara Ingold Heppner, Berlin Christian Jackisch, Offenbach Wolfgang Janni, Ulm Panagiotis Kapetas, Vienna Stefanie Kemper, Münster Peter Kern, Essen Julia Knaudt, Mannheim Dieter Köberle, Basel Katja Krauss, Aachen Hans H. Kreipe, Hanover Robert Krempien, Berlin Marco Krengli, Novara Annette Lebeau, Hamburg Cornelia Liedtke, Lübeck Bettina Loehrs, Landshut Michael P. Lux, Erlangen Heinrich Magometschnigg, Vienna Anthony Maxwell, Manchester Brigitte Mlineritsch, Salzburg Volkmar Müller, Hamburg Hans Neubauer, Düsseldorf Olaf Ortmann, Regensburg Stephanie Otto, Ulm Shani Paluch-Shimon, Tel Hashomer Montserrat Pazos, Munich Edgar Petru, Graz
Georg Pfeiler, Vienna

Gunda Pristauz-Telsnigg, Graz

Isabel Radke, Münster

Toralf Reimer, Rostock

Roland Reitsamer, Salzburg

Alexandra Resch, Vienna

Joana Ribeiro, Lisbon

Karin Ribi, Berne

Johanna Ringwald, Tübingen

Gabriel Rinnerthaler, Salzburg

Achim Rody, Lübeck

Eugen Ruckhäberle, Düsseldorf

Anton Scharl, Amberg

Christian Schem, Kiel

Barbara Schmalfeldt, Hamburg

Seraina Schmid, Grabs

Marcus Schmidt, Mainz

Thorsten Schmidt, Kiel

Rita K. Schmutzler, Cologne

Peter Sinn, Heidelberg

Atilla Soran, Pittsburgh, PA

Eliane Taube, Berlin

Hans Tesch, Frankfurt/M.

Daniela Trog, Münster

Christoph Uleer, Hildesheim

S. Weigel, Münster

Stefan Welz, Tübingen

Ludwig Wildt, Innsbruck Isabell Witzel, Hamburg

Ramona Woitek, Vienna

Rachel Würstlein, Munich

Bobby Zachariae, Aarhus

Eva Zopf, Melbourne

\section{KARGER}

